\title{
FRACTIONAL INTEGRAL OPERATORS IN NONHOMOGENEOUS SPACES
}

\author{
H. GUNAWAN ${ }^{\bowtie}$, Y. SAWANO and I. SIHWANINGRUM
}

(Received 3 November 2008)

\begin{abstract}
We discuss here the boundedness of the fractional integral operator $I_{\alpha}$ and its generalized version on generalized nonhomogeneous Morrey spaces. To prove the boundedness of $I_{\alpha}$, we employ the boundedness of the so-called maximal fractional integral operator $I_{a, k}^{*}$. In addition, we prove an Olsentype inequality, which is analogous to that in the case of homogeneous type.
\end{abstract}

2000 Mathematics subject classification: primary 42B20; secondary 42B35, 47G10, 31B10, $26 \mathrm{~A} 33$.

Keywords and phrases: fractional integral operators, generalized Morrey spaces, nondoubling measures, Olsen's inequality.

\section{Introduction}

Let $\mathbb{R}^{d}$ be equipped with a (positive) Radon measure $\mu$ satisfying the growth condition:

$$
\mu\left(Q\left(x, \ell_{Q}\right)\right) \leq C \cdot\left(\ell_{Q}\right)^{n}, \quad x \in \operatorname{supp} \mu, \ell_{Q}>0,
$$

for some constants $C$ and $n$, where $0<n \leq d$. Here $Q\left(x, \ell_{Q}\right)$ denotes the cube with centre $x \in \mathbb{R}^{d}$ and side length $\ell_{Q}$. Many authors call such $\mu$ a nondoubling measure, since it does not necessarily satisfy the so-called doubling condition, a condition which is usually required when one studies the properties of an operator in a measure space. In this case, the space $\left(\mathbb{R}^{d}, \mu\right)$ is known as a nonhomogeneous space. See, for instance, the works of $[11,12,18,20]$.

In this paper, we study the fractional integral operator $I_{\alpha}=I_{\alpha}(n, \mu)$, given by the formula

$$
I_{\alpha} f(x):=\int_{\mathbb{R}^{d}} \frac{f(y)}{|x-y|^{n-\alpha}} d \mu(y),
$$

where $0<\alpha<n$, and its generalized version (which we define later). Note that if $n=d$ and $\mu$ is the usual Lebesgue measure on $\mathbb{R}^{d}$, then $I_{\alpha}$ is the classical fractional integral operator which is known to be bounded from $L^{p}$ to $L^{q}$ for $1<p<d / \alpha$ 
and $1 / q=1 / p-\alpha / d$; this is due to Hardy and Littlewood [6, 7] and Sobolev [16]. Some extensions of this result may be found in, for instance, [1, 9], or in [17, 19], and the references therein.

In the nonhomogeneous setting, the boundedness of $I_{\alpha}$ on the Lebesgue spaces $L^{p}(\mu)$ has been studied recently in $[4,15]$. Our goal here is to prove the boundedness of $I_{\alpha}$, and its generalized version, on the generalized nonhomogeneous Morrey space $\mathcal{M}_{p, \phi}(k, \mu)$. In addition, we also prove an Olsen-type inequality involving $I_{\alpha}$ and a multiplicator $W$ (see [13]).

Before we go into details, let us fix some notation. By $\mathcal{Q}(\mu)$ we mean the set of all cubes with positive $\mu$-measure. Given $Q \in \mathcal{Q}(\mu)$ and $k>1$, let us set $k Q$ as the cube concentric to $Q$ and having $k$-times as long side length as $Q$. Denoting by $\ell_{Q}$ the side length of $Q \in \mathcal{Q}(\mu)$, we have $\ell_{k} Q=k \ell_{Q}$. Next, we write $\|f: X\|$ to denote the norm of $f$ in the space $X$. The generalized nonhomogeneous Morrey spaces $\mathcal{M}_{p, \phi}(k, \mu)$ is the set of all $\mu$-locally integrable functions $f$ on $\mathbb{R}^{d}$ for which

$$
\left\|f: \mathcal{M}_{p, \phi}(k, \mu)\right\|:=\sup _{Q} \frac{1}{\phi(\mu(k Q))}\left(\frac{1}{\mu(k Q)} \int_{Q}|f(y)|^{p} d \mu(y)\right)^{1 / p}<\infty .
$$

Here $1<p<\infty$ and we always assume tacitly that the function $\phi:(0, \infty) \rightarrow(0, \infty)$ is almost decreasing, that is, there exists $C_{1}>0$ such that $\phi(s) \geq C_{1} \phi(t)$ for $s<t$; and also satisfies the doubling condition, that is, there exists $C_{2}>0$ such that

$$
\frac{1}{C_{2}} \leq \frac{\phi(s)}{\phi(t)} \leq C_{2}
$$

whenever $1 \leq s / t \leq 2$. Meanwhile, $k>1$ serves as an auxiliary parameter.

As in [14], we have the following fact.

FACT 1 . For $k_{1}, k_{2}>1$, the spaces $\mathcal{M}_{p, \phi}\left(k_{1}, \mu\right)$ and $\mathcal{M}_{p, \phi}\left(k_{2}, \mu\right)$ coincide as a set and their norms are mutually equivalent.

In view of this fact, we set $\mathcal{M}_{p, \phi}(\mu):=\mathcal{M}_{p, \phi}(2, \mu)$. One may observe that if $1 \leq p_{1} \leq p_{2}<\infty$, then $\mathcal{M}_{p_{2}, \phi}(\mu) \subseteq \mathcal{M}_{p_{1}, \phi}(\mu)$ for we have $\left\|f: \mathcal{M}_{p_{1}, \phi}\right\| \leq \| f:$ $\mathcal{M}_{p_{2}, \phi} \|$ whenever $f \in \mathcal{M}_{p_{2}, \phi}$.

To prove the boundedness of $I_{\alpha}$ from $\mathcal{M}_{p, \phi}(\mu)$ to $\mathcal{M}_{q, \psi}(\mu)$, we need to employ the (modified) maximal operator $M_{k}(k>1)$, which is defined by

$$
M_{k} f(x):=\sup _{Q \ni x} \frac{1}{\mu(k Q)} \int_{Q} f(y) d \mu(y),
$$

for all $\mu$-locally integrable functions $f$ on $\mathbb{R}^{d}$.

Theorem 1.1 (Sawano [14]). For $p>1, M_{k}$ is a bounded operator on $\mathcal{M}_{p, \phi}(\mu)$. 


\section{The boundedness of $I_{\alpha}$}

To study the boundedness of $I_{\alpha}$ on the generalized Morrey spaces, we define the maximal fractional integral operator $I_{a, \kappa}^{*}$, given by

$$
I_{a, \kappa}^{*} f(x):=\int_{\mathbb{R}^{d}} K_{a, \kappa}^{*}(x, y) f(y) d \mu(y),
$$

where the kernel $K_{a, \kappa}^{*}$ is given by

$$
K_{a, \kappa}^{*}(x, y):=\sup _{Q \ni x, y} \mu(\kappa Q)^{a-1} .
$$

Here $0<a<1$ and $\kappa>1$; both of them serve as parameters. Since we are not concerned with keeping track of the precise value of various constants, we denote by $C$ various constants independent of functions $f$. Using the following fact, it suffices for us to study the boundedness of $I_{\alpha / n, \kappa}^{*}$.

FACT 2 (Sawano et al. [15]). We have $I_{\alpha} f \leq C I_{\alpha / n, \kappa}^{*} f$ for all positive $\mu$-measurable functions $f$.

Our result is the following theorem, which is in some sense stronger than the result obtained in [14].

THEOREM 2.1. Let $a=\alpha / n \in(0,1)$. If the function $\phi$ is surjective and satisfies the inequality $\phi(t) \leq C t^{b}$ with $-1 / p \leq b<-a<0$, then $I_{a, \kappa}^{*}$ is bounded from $\mathcal{M}_{p, \phi}(\mu)$ to $\mathcal{M}_{q, \phi^{p / q}}(\mu)$, where $p>1$ and $q=b p /(a+b)$.

A helpful remark may be in order. In the above theorem, the hypothesis $b \geq-1 / p$ is needed for $\left\|f: \mathcal{M}_{p, \phi}(\mu)\right\|$ to make sense. Meanwhile, the hypothesis $b<-a$ together with $p / q=(a+b) / b<1$ implies that $p<q$. When $\mu$ is the usual Lebesgue measure on $\mathbb{R}^{d}$ and $\phi(t)=t^{(\lambda-n) /(n p)}$ with $0 \leq \lambda<n-\alpha p$ and $1<p<n / \alpha$, the above results recover the boundedness of $I_{\alpha}$ from the classical Morrey space $L^{p, \lambda}$ to $L^{q, \lambda}$ where $1 / q=1 / p-\alpha /(n-\lambda)$ (see [1]). For related results in the generalized 'homogeneous' Morrey spaces, see [3,5].

Proof of TheOREM 2.1. Without loss of generality, we assume that $f \geq 0$, not identically zero, and a usual truncation procedure allows us to assume even that $M_{\sqrt{\kappa}} f(x)$ is finite everywhere. For $x \in \operatorname{supp} \mu$ and $\tau>0$, we write

$$
A_{j}:=\left\{y \in \mathbb{R}^{d} \backslash\{x\}: 2^{j-1} \tau<\inf _{Q \ni x, y} \mu(\kappa Q) \leq 2^{j} \tau\right\},
$$

for every $j \in \mathbb{Z}$. As in [15], there exists a collection of cubes $Q_{j}^{1}, \ldots, Q_{j}^{N_{\kappa}}$ containing $x$, with the covering constant $N_{\kappa}$ being independent of $x, \tau$, and $j$, such that

$$
A_{j} \subseteq \sqrt{\kappa} Q_{j}^{1} \cup \cdots \cup \sqrt{\kappa} Q_{j}^{N_{\kappa}}
$$

and

$$
\mu\left(\kappa Q_{j}^{l}\right) \leq 2^{j+1} \tau, \quad 1 \leq l \leq N_{\kappa},
$$

for every $j \in \mathbb{Z}$. As a special case we accept $Q_{j}^{1}=\mathbb{R}^{d}$, although $\mathbb{R}^{d}$ is not compact. 
In the following, for the sake of simplicity, we assume that the $Q_{j}^{l}$ are all compact. (If this is not the case, a minor modification suffices.)

Now, for each $y \in A_{j}$, we have $K_{a, \kappa}^{*}(x, y)<C\left(2^{j} \tau\right)^{a-1}$, and so

$$
\begin{aligned}
I_{a, \kappa}^{*} f(x) & \leq C \sum_{j \in \mathbb{Z}}\left(2^{j} \tau\right)^{a-1} \int_{A_{j}} f(y) d \mu(y) \\
& \leq C \sum_{j \in \mathbb{Z}} \sum_{l=1}^{N_{\kappa}}\left(2^{j} \tau\right)^{a-1} \int_{\sqrt{\kappa} Q_{j}^{l}} f(y) d \mu(y) \\
& \leq C\left[\sum_{j=-\infty}^{0} \sum_{l=1}^{N_{\kappa}} \cdots+\sum_{j=1}^{\infty} \sum_{l=1}^{N_{\kappa}} \cdots\right] \\
& =: C[I+I I] .
\end{aligned}
$$

For the first summation, we use the definition of $M_{\sqrt{\kappa}} f$ to have

$$
\begin{aligned}
I & \leq C \sum_{j=-\infty}^{0} \sum_{l=1}^{N_{\kappa}}\left(2^{j} \tau\right)^{a}\left(\frac{1}{\mu\left(\kappa Q_{j}^{l}\right)} \int_{\sqrt{\kappa} Q_{j}^{l}} f(y) d \mu(y)\right) \\
& \leq C \tau^{a} M_{\sqrt{\kappa}} f(x) .
\end{aligned}
$$

For the second summation, we make use of Hölder's inequality to obtain

$$
\begin{aligned}
I I & \leq \sum_{j=1}^{\infty} \sum_{l=1}^{N_{\kappa}}\left(2^{j} \tau\right)^{a-1}\left(\mu\left(\kappa Q_{j}^{l}\right)\right)^{1-(1 / p)}\left(\int_{\sqrt{\kappa} Q_{j}^{l}} f(y)^{p} d \mu(y)\right)^{1 / p} \\
& \leq \sum_{j=1}^{\infty} \sum_{l=1}^{N_{\kappa}}\left(2^{j} \tau\right)^{a}\left(\frac{1}{\mu\left(\kappa Q_{j}^{l}\right)} \int_{\sqrt{\kappa} Q_{j}^{l}} f(y)^{p} d \mu(y)\right)^{1 / p} \\
& \leq \sum_{j=1}^{\infty} \sum_{l=1}^{N_{\kappa}}\left(2^{j} \tau\right)^{a} \phi\left(\mu\left(\kappa Q_{j}^{l}\right)\right)\left\|f: \mathcal{M}_{p, \phi}(\mu)\right\| \\
& \leq C\left\|f: \mathcal{M}_{p, \phi}(\mu)\right\| \sum_{j=1}^{\infty}\left(2^{j} \tau\right)^{a}\left(\mu\left(\kappa Q_{j}^{l}\right)\right)^{b} \\
& \leq C\left\|f: \mathcal{M}_{p, \phi}(\mu)\right\| \sum_{j=1}^{\infty}\left(2^{j} \tau\right)^{a+b} \\
& \leq C \tau^{a+b}\left\|f: \mathcal{M}_{p, \phi}(\mu)\right\|
\end{aligned}
$$

since $b<-a$.

Combining the two estimates and choosing $\tau=\left(M_{\sqrt{\kappa}} f(x) /\left\|f: \mathcal{M}_{p, \phi}(\mu)\right\|\right)^{1 / b}$, we obtain

$$
\begin{aligned}
I_{a, \kappa}^{*} f(x) & \leq C\left(M_{\sqrt{\kappa}} f(x)\right)^{(a / b)+1}\left\|f: \mathcal{M}_{p, \phi}(\mu)\right\|^{-(a / b)} \\
& \leq C\left(M_{\sqrt{\kappa}} f(x)\right)^{p / q}\left\|f: \mathcal{M}_{p, \phi}(\mu)\right\|^{1-(p / q)} .
\end{aligned}
$$


Since $M_{\sqrt{\kappa}}$ is bounded on $\mathcal{M}_{p, \phi}(\mu)$, we obtain

$$
\left\|I_{a, \kappa}^{*} f: \mathcal{M}_{q, \phi^{p / q}}(\mu)\right\| \leq C\left\|f: \mathcal{M}_{p, \phi}(\mu)\right\|,
$$

that is, $I_{a, \kappa}^{*}$ is bounded from $\mathcal{M}_{p, \phi}(\mu)$ to $\mathcal{M}_{q, \phi^{p / q}}(\mu)$, as desired.

Corollary 2.2. If $\phi, p$ and $q$ are as in Theorem 2.1, then $I_{\alpha}$ is bounded from $\mathcal{M}_{p, \phi}(\mu)$ to $\mathcal{M}_{q, \phi^{p / q}}(\mu)$.

REMARK 2.3. As a consequence of our result, we have that if $T$ is a kernel operator with kernel $K$ satisfying

$$
K(x, y) \leq C|x-y|^{\alpha-n},
$$

then $T$ is bounded from $\mathcal{M}_{p, \phi}(\mu)$ to $\mathcal{M}_{q, \phi^{p / q}}(\mu)$, where $\phi, p$ and $q$ are as in Theorem 2.1. With this result, one may also obtain estimates for fractional powers of operators that generate semigroups with the Gaussian bounds for the associated heat kernels (see [2] about heat kernels).

2.1. An Olsen-type inequality Following Corollary 2.2, we have the Olsen-type inequality below. (For its origin, see the work of Olsen [13]. For an analogous result in the 'homogeneous' case, see [5]. The 'Olsen inequality' is useful in understanding the behaviour of the solution to a perturbed Schrödinger equation with a small perturbed potential $W$; see [8].)

Corollary 2.4. If $\phi, p$ and $q$ are as in Theorem 2.1, then $W \cdot I_{\alpha}$ is bounded on $\mathcal{M}_{p, \phi}(\mu)$ provided that $W \in \mathcal{M}_{s, \phi^{p / s}}(\mu)$, where $1 / s=1 / p-1 / q$. Precisely, we have

$$
\left\|W \cdot I_{\alpha} f: \mathcal{M}_{p, \phi}(\mu)\right\| \leq C\left\|W: \mathcal{M}_{s, \phi^{p / s}}(\mu)\right\| \cdot\left\|f: \mathcal{M}_{p, \phi}(\mu)\right\|,
$$

where $1 / s=1 / p-1 / q$.

The following theorem provides an Olsen-type inequality for a multiplication operator involving the fractional integral operator $I_{a, \kappa}^{*}$.

THEOREM 2.5. Suppose that $t^{1 / p} \phi(t)$ is almost increasing and satisfies the inequality

$$
\int_{r}^{\infty} t^{a-1} \phi(t) d t \leq C r^{a} \phi(r)
$$

for all $r>0$ with some $a<1 / p$ and $C>0$ independent of $r$. Then

$$
\left\|W \cdot I_{a, \kappa}^{*} f: \mathcal{M}_{p, \phi}(\mu)\right\| \leq C\left\|W: L^{1 / a}(\mu)\right\| \cdot\left\|f: \mathcal{M}_{p, \phi}(\mu)\right\| .
$$

Proof 1. Let $Q \in \mathcal{Q}(\mu)$ be fixed. We shall establish

$$
I \leq C\left\|W: L^{1 / a}(\mu)\right\| \cdot\left\|f: \mathcal{M}_{p, \phi}(\mu)\right\|
$$


with $K$ sufficiently large. We decompose $f$ according to $K Q$, where

$$
I:=\frac{1}{\phi(\mu(2 K Q))}\left(\frac{1}{\mu(2 K Q)} \int_{Q}\left|W \cdot I_{a, \kappa}^{*} f(y)\right|^{p} d \mu(y)\right)^{1 / p} .
$$

Set $f_{1}=f \cdot \chi_{K} Q$ and $f_{2}=f-f_{1}$. Along this decomposition, (2.2) can be split into

$$
\begin{gathered}
I I \leq C\left\|W: L^{1 / a}(\mu)\right\| \cdot\left\|f: \mathcal{M}_{p, \phi}(\mu)\right\| \\
I I I \leq C\left\|W: L^{1 / a}(\mu)\right\| \cdot\left\|f: \mathcal{M}_{p, \phi}(\mu)\right\|,
\end{gathered}
$$

where

$$
\begin{aligned}
I I & :=\frac{1}{\phi(\mu(2 K Q))}\left(\frac{1}{\mu(2 K Q)} \int_{Q}\left|W \cdot I_{a, \kappa}^{*} f_{1}(y)\right|^{p} d \mu(y)\right)^{1 / p} \\
I I I & :=\frac{1}{\phi(\mu(2 K Q))}\left(\frac{1}{\mu(2 K Q)} \int_{Q}\left|W \cdot I_{a, \kappa}^{*} f_{2}(y)\right|^{p} d \mu(y)\right)^{1 / p} .
\end{aligned}
$$

As for (2.3) we use Hölder's inequality to obtain

$$
\begin{aligned}
I I & \leq \frac{1}{\phi(\mu(2 K Q))}\left(\frac{1}{\mu(2 K Q)} \int_{\mathbb{R}^{d}}\left|W \cdot I_{a, \kappa}^{*} f_{1}(y)\right|^{p} d \mu(y)\right)^{1 / p} \\
& \leq \frac{C}{\phi(\mu(2 K Q))}\left(\frac{1}{\mu(2 K Q)}\right)^{1 / p}\left\|W: L^{1 / a}(\mu)\right\| \cdot\left\|f_{1}: L^{p}(\mu)\right\| \\
& \leq C\left\|W: L^{1 / a}(\mu)\right\| \cdot\left\|f: \mathcal{M}_{p, \phi}(\mu)\right\| .
\end{aligned}
$$

Let us denote by $c_{Q}$ the centre of $Q$. To obtain (2.4), we observe that

$$
K_{a, \kappa}^{*}(x, y) \leq \sup _{c_{Q}, y \in R \in \mathcal{Q}(\mu)} \mu(\sqrt{\kappa} R)^{-1+a}
$$

for all $x \in Q$ and $y \in \mathbb{R}^{d} \backslash K Q$, provided $K$ is sufficiently large. Therefore, it suffices to show

$$
I V \leq C\left\|W: L^{1 / a}(\mu)\right\| \cdot\left\|f: \mathcal{M}_{p, \phi}(\mu)\right\|,
$$

where

$$
\begin{aligned}
I V & :=\frac{1}{\phi(\mu(2 K Q))}\left(\frac{1}{\mu(2 K Q)} \int_{Q}\left|W \cdot \tilde{I}_{a}^{L} f_{2}\right|^{p} d \mu\right)^{1 / p} \\
\tilde{I}_{a}^{L} g(x) & :=\int_{\mathbb{R}^{d}} \tilde{k}_{a}^{L}(x, y) f(y) d \mu(y) \\
\tilde{k}_{a}^{L}(x, y) & :=\sup _{\substack{Q \subset R \in \mathcal{Q}(\mu) \\
\ell_{R} \leq L, y \in R}} \mu(\sqrt{\kappa} R)^{-1+a} .
\end{aligned}
$$


Let us set

$$
A_{j}:=\left\{y \in \operatorname{supp} \mu: 2^{j-1}<\inf _{\substack{Q \subset R \in \mathcal{Q}(\mu) \\ \ell_{R} \leq L, y \in R}} \mu(\sqrt{\kappa} R) \leq 2^{j}\right\}
$$

Then from the definition of $\tilde{k}_{a}^{L}$ for each $j \in \mathbb{Z}$, a geometric observation yields a finite collection of cubes $Q_{j}^{1}, \ldots, Q_{j}^{N_{\kappa}} \in \mathcal{Q}(\mu)$ such that

$$
A_{j} \subset \bigcup_{l=1}^{N_{\kappa}} \sqrt[4]{\kappa} Q_{j}^{l}
$$

and that

$$
2^{j-1} \leq \mu\left(\sqrt{\kappa} Q_{j}^{l}\right) \leq 2^{j} \quad \text { for each } l=1, \ldots, N_{\kappa} .
$$

Here the number $N_{\kappa}$ is again a covering constant which may depend only on $\kappa$ and $d$. Again it can happen that the cube $Q_{j}^{1}$ equals $\mathbb{R}^{d}$. In this case we only need to make a minor modification of the proof below.

With the help of this covering we obtain

$$
\begin{aligned}
\tilde{I}_{a}^{L} f_{2}(x) & \leq C \sum_{\substack{j \in \mathbb{Z} \\
A_{j} \neq \emptyset}} \sum_{l=1}^{N_{\kappa}} 2^{-(1-a) j} \int_{\sqrt[4]{\kappa} Q_{j}^{l}} f(y) d \mu(y) \\
& \leq C \sum_{\substack{j \in \mathbb{Z} \\
A_{j} \neq \emptyset}} 2^{a j} \phi\left(2^{j}\right)\left\|f: \mathcal{M}_{1, \phi}(\mu)\right\| .
\end{aligned}
$$

Note that if $A_{j} \neq \emptyset$, then we have $\mu(Q) \leq 2^{j}$. As a consequence, we have by virtue of (2.1)

$$
\begin{aligned}
\sum_{\substack{j \in \mathbb{Z} \\
A_{j} \neq \emptyset}} 2^{a j} \phi\left(2^{j}\right) & \leq C \sum_{\substack{j \in \mathbb{Z} \\
A_{j} \neq \emptyset}} \int_{2^{j}}^{2^{j+1}} t^{a-1} \phi(t) d t \\
& \leq C \int_{\mu(Q)}^{\infty} t^{a-1} \phi(t) d t \\
& \leq C \mu(Q)^{a} \phi(\mu(Q)) .
\end{aligned}
$$

From this inequality and the fact that $\left\|f: \mathcal{M}_{1, \phi}(\mu)\right\| \leq\left\|f: \mathcal{M}_{p, \phi}(\mu)\right\|$, we now have

$$
\tilde{I}_{a}^{L} f_{2}(x) \leq C \mu(Q)^{a} \phi(\mu(Q))\left\|f: \mathcal{M}_{p, \phi}(\mu)\right\|
$$


for all $x \in Q$ and, hence,

$$
\begin{aligned}
I V & \leq C \frac{\mu(Q)^{a} \phi(\mu(Q))\left(\int_{Q}|W(y)|^{p} d \mu(y)\right)^{1 / p}}{\mu(2 K Q)^{1 / p} \phi(\mu(2 K Q))}\left\|f: \mathcal{M}_{p, \phi}(\mu)\right\| \\
& \leq C \frac{\mu(Q)^{1 / p} \phi(\mu(Q))\left(\int_{Q}|W(y)|^{1 / a} d \mu(y)\right)^{a}}{\mu(2 K Q)^{1 / p} \phi(\mu(2 K Q))}\left\|f: \mathcal{M}_{p, \phi}(\mu)\right\| \\
& \leq C\left(\int_{\mathbb{R}^{d}}|W(y)|^{1 / a} d \mu(y)\right)^{a}\left\|f: \mathcal{M}_{p, \phi}(\mu)\right\|,
\end{aligned}
$$

since $t^{1 / p} \phi(t)$ is almost increasing. This is the desired result.

\section{A generalized version}

The generalized fractional integral operator $T_{\rho}$, defined for a given function $\rho$ : $(0, \infty) \rightarrow(0, \infty)$ by

$$
T_{\rho} f(x)=\int_{\mathbb{R}^{d}} \frac{\rho(|x-y|)}{|x-y|^{d}} f(y) d y,
$$

was first studied by $[9,10]$, in the homogeneous setting. For $\rho(t)=t^{\alpha}, 0<\alpha<d$, the operator $T_{\rho}$ is equal to $I_{\alpha}$, the classical fractional integral operator.

Now, we discuss an analogue of $T_{\rho}$ in the nonhomogeneous setting. Write

$$
D(x, y):=\inf _{x, y \in Q \in \mathcal{Q}(\mu)} \mu(\kappa Q)
$$

and

$$
l_{\rho}(x, y):=\frac{\rho(D(x, y))}{D(x, y)} .
$$

Given a positive function $f$, we define $T_{\rho}$ by

$$
T_{\rho} f(x):=\int_{\mathbb{R}^{d}} l_{\rho}(x, y) f(y) d \mu(y) .
$$

Note that for $\rho(t)=t^{\alpha}$, the operator $T_{\rho}$ takes the form of $I_{a, \kappa}^{*}$.

THEOREM 3.1. Let $1<p<q<\infty$. Assume that $\phi$ is surjective and $\rho$ satisfies the doubling condition. Suppose further that there exists $C>0$ such that

$$
\phi(r) \int_{0}^{r} \frac{\rho(t)}{t} d t+\int_{r}^{\infty} \frac{\phi(t) \rho(t)}{t} d t \leq C \phi(r)^{p / q}
$$

for every $r>0$. Then, there exists $C>0$ such that

$$
\left\|T_{\rho} f: \mathcal{M}_{q, \phi^{p / q}}(\mu)\right\| \leq C\left\|f: \mathcal{M}_{p, \phi}(\mu)\right\|
$$

for all positive $f \in \mathcal{M}_{p, \phi}(\mu)$. 
PROOF 2. The kernel being positive, we can truncate the kernel with respect to the side length of cubes. Let $L>0$ and define

$$
l_{\rho}^{L}(x, y):=\sup _{\substack{x, y \in Q \in \mathcal{Q}(\mu) \\ \ell_{Q} \leq L}} \frac{\rho(\mu(\kappa Q))}{\mu(\kappa Q)} .
$$

Given a positive function $f$, define

$$
T_{\rho}^{L} f(x):=\int_{\mathbb{R}^{d}} l_{\rho}^{L}(x, y) f(y) d \mu(y) .
$$

It suffices to prove the assertion for $T_{\rho}^{L}$ instead of $T_{\rho}$ with constants independent of $L$. Fix $x \in \operatorname{supp} \mu$ and $\tau>0$. For $j \in \mathbb{Z}$ we define

$$
A_{j}:=\left\{y \in \mathbb{R}^{d}: 2^{j-1} \tau<\inf _{\substack{x, y \in Q \in \mathcal{Q}(\mu) \\ \ell Q \leq L}} \mu(\kappa Q) \leq 2^{j} \tau\right\} .
$$

Then

$$
T_{\rho}^{L} f(x) \leq C \sum_{j \in \mathbb{Z}} \frac{\rho\left(2^{j} \tau\right)}{2^{j} \tau} \int_{A_{j}} f(y) d \mu(y) .
$$

As before, there exists $N_{\kappa}$ with the following properties

$$
A_{j} \subset \sqrt{\kappa} Q_{j}^{1} \cup \cdots \cup \sqrt{\kappa} Q_{j}^{N_{\kappa}} .
$$

Therefore,

$$
\begin{aligned}
T_{\rho}^{L} f(x) \leq & C \sum_{j \in \mathbb{Z}} \sum_{l=1}^{N_{\kappa}} \frac{\rho\left(2^{j} \tau\right)}{2^{j} \tau} \int_{\sqrt{\kappa} Q_{j}^{l}} f(y) d \mu(y) \\
\leq & C \sum_{j \in \mathbb{Z} \backslash \mathbb{N}} \sum_{l=1}^{N_{\kappa}} \rho\left(2^{j} \tau\right) \cdot M_{\sqrt{\kappa}} f(x) \\
& \quad+C \sum_{j \in \mathbb{N}} \sum_{l=1}^{N_{\kappa}} \frac{\rho\left(2^{j} \tau\right)}{\left(2^{j} \tau\right)^{1 / p}}\left(\int_{\sqrt{\kappa} Q_{j}^{l}} f(y)^{p} d \mu(y)\right)^{1 / p} \\
\leq C & \int_{0}^{\tau} \frac{\rho(t)}{t} d t \cdot M_{\sqrt{\kappa}} f(x)+C \int_{\tau}^{\infty} \frac{\rho(t) \phi(t)}{t} d t \cdot\left\|f: \mathcal{M}_{p, \phi}(\mu)\right\| \\
\leq C & (\tau)^{\frac{p}{q}-1} M_{\sqrt{\kappa}} f(x)+\phi(\tau)^{p / q}\left\|f: \mathcal{M}_{p, \phi}(\mu)\right\| .
\end{aligned}
$$

Assuming $\phi$ surjective and that $1<p<q<\infty$, we can optimize the above inequality. As a consequence

$$
T_{\rho}^{L} f(x) \leq C\left(M_{\sqrt{\kappa}} f(x)\right)^{p / q} \cdot\left\|f: \mathcal{M}_{p, \phi}(\mu)\right\|^{1-p / q} .
$$

Now that the maximal operator $M_{\sqrt{\kappa}}$ is bounded on $\mathcal{M}_{p, \phi}(\mu)$, we have the desired result. 
COROllary 3.2. Suppose that $\phi$ is surjective. If $\rho$ satisfies the doubling condition and

$$
\phi(r) \int_{0}^{r} \frac{\rho(t)}{t} d t+\int_{r}^{\infty} \frac{\phi(t) \rho(t)}{t} d t \leq C \phi(r)^{p / q}
$$

for $1<p<q<\infty$, then

$$
\left\|W \cdot T_{\rho} f: \mathcal{M}_{p, \phi}(\mu)\right\| \leq C\left\|W: \mathcal{M}_{s, \phi^{p / s}}(\mu)\right\| \cdot\left\|f: \mathcal{M}_{p, \phi}(\mu)\right\|
$$

provided that $W \in \mathcal{M}_{s, \phi^{p / s}}$ where $1 / s=1 / p-1 / q$.

\section{Acknowledgements}

H. Gunawan and I. Sihwaningrum are supported by Fundamental Research Program 2008-2009. Y. Sawano is supported by Research Fellowships of the Japan Society for the Promotion of Science for Young Scientists. All authors are very grateful to Professor E. Nakai for his information about the operator $T_{\rho}$. Special thanks also go to the anonymous referee for their comments on the earlier version of this paper. (In particular, the remark after Corollary 2.2 is added according to their suggestion.)

\section{References}

[1] D. Adams, 'A note on Riesz potentials', Duke Math. J. 42 (1975), 765-778.

[2] E. B. Davies, Heat Kernels and Spectral Theory (Cambridge University Press, Cambridge, 1989).

[3] Eridani, H. Gunawan and E. Nakai, 'On generalized fractional integral operators', Sci. Math. Jpn. 60 (2004), 539-550.

[4] J. García-Cuerva and E. Gatto, 'Boundedness properties of fractional integral operators associated to non-doubling measures', Studia Math. 162 (2004), 245-261.

[5] H. Gunawan and Eridani, 'Fractional integrals and generalized Olsen inequalities', Kyungpook Math. J. 49 (2009), 31-39.

[6] G. H. Hardy and J. E. Littlewood, 'Some properties of fractional integrals. I', Math. Z. 27 (1927), 565-606.

[7] - 'Some properties of fractional integrals. II', Math. Z. 34 (1932), 403-439.

[8] K. Kurata, S. Nishigaki and S. Sugano, 'Boundedness of integral operators on generalized Morrey spaces and its application to Schrödinger operators', Proc. Amer. Math. Soc. 128 (2002), $1125-1134$.

[9] E. Nakai, 'On generalized Fractional integrals in the Orlicz spaces on spaces of homogeneous type', Sci. Math. Jpn. 54 (2001), 473-487 (e4: 901-915).

[10] E. Nakai and H. Sumitomo, 'On generalized Riesz potentials and spaces of some smooth functions', Sci. Math. Jpn. 54 (2001), 463-472 (e4: 891-901).

[11] F. Nazarov, S. Treil and A. Volberg, 'Weak type estimates and Cotlar inequalities for CalderónZygmund operators on nonhomogeneous spaces', Internat. Math. Res. Notices 9 (1998), 463-487.

[12] , 'The Tb-theorem on non-homogeneous spaces', Acta Math. 190 (2003), 151-239.

[13] P. A. Olsen, 'Fractional integration, Morrey spaces and a Schrödinger equation', Comm. Partial Differential Equations 20 (1995), 2005-2055.

[14] Y. Sawano, 'Generalized Morrey spaces for non-doubling measures', Non-linear Differential Equations Appl. 15 (2008), 413-425.

[15] Y. Sawano, T. Sobukawa and H. Tanaka, 'Limiting case of the boundedness of fractional integral operators on non-homogeneous space', J. Inequal. Appl. (2006), Art. ID 92470, 16pp.

[16] S. L. Sobolev, 'On a theorem in functional analysis' (in Russian), Mat. Sb. 46 (1938), 471-497. (English translation in Amer. Math. Soc. Transl. Ser. (2) 34 (1963), 39-68.) 
[17] E. M. Stein, Singular Integrals and Differentiability Properties of Functions (Princeton University Press, Princeton, NJ, 1970).

[18] X. Tolsa, 'BMO, $H^{1}$, and Calderón-Zygmund operators for non doubling measures', Math. Ann. 319 (2001), 89-149.

[19] A. Torchinsky, Real-variable Methods in Harmonic Analysis (Academic Press, New York, 1986).

[20] J. Verdera, 'The fall of the doubling condition in Calderón-Zygmund theory', Publ. Mat. (2002), 275-292 (Special Volume).

H. GUNAWAN, Department of Mathematics, Bandung Institute of Technology, Bandung 40132, Indonesia

e-mail: hgunawan@math.itb.ac.id

Y. SAWANO, Department of Mathematics, Gakushuin University, 1-5-1 Mejiro, Toshima-ku, Tokyo 171-8588, Japan

e-mail: yosihiro@math.gakushuin.ac.jp

I. SIHWANINGRUM, Department of Mathematics, Bandung Institute of Technology, Bandung 40132, Indonesia

e-mail: hanidha@students.itb.ac.id

and

Permanent address: Department of Mathematics, General Soedirman University, Purwokerto 53122, Indonesia 